\title{
FRACTURA SUBCAPITAL DE FÉMUR SECUNDARIA A OSTEOPO- ROSIS IDIOPÁTICA EN EL EMBARAZO: A PROPÓSITO DE UN CASO
}

\section{Constanza Caliendo ${ }^{1}$, Ana María Fernández Alonso. ${ }^{1}$, Milagros Cruz Martínez. ${ }^{1}$, Amelia Vizcaíno Martínez. ${ }^{1}$, Ana Rosa Blázquez Ruiz. ${ }^{1}$}

${ }^{1}$ Servicio de Obstetricia y Ginecología, Hospital Universitario San Cecilio, Granada, España.

\section{RESUMEN}

Presentamos el caso de una paciente imposibilitada para la deambulación durante el puerperio, consecuencia de una fractura de fémur producida por una osteoporosis idiopática durante el embarazo. A los 11 meses del parto, la paciente presenta una evolución favorable con tratamiento médico con bifosfonatos y calcio.

\section{PALABRAS CLAVE: Osteoporosis, embarazo, puerperio, fractura femoral}

\section{SUMMARY}

We present the case of a mobility disabled person during puerperium as a consequence of a femur fracture due to an idiopathic osteoporosis during pregnancy. Eleven months after delivery, the patient's evolution was favourable with a medical treatment using bisphosphonates and calcium.

KEY WORDS: Osteoporosis, pregnancy, postpartum period, femoral fracture

\section{INTRODUCCIÓN}

Durante el embarazo pueden ocurrir cambios de la densidad mineral ósea, pudiéndose llegar a producir una osteoporosis transitoria. Se ha descrito una mayor incidencia de ésta durante el tercer trimestre del embarazo y raramente se puede complicar con una fractura de cabeza femoral (1). El objetivo de esta comunicación es presentar un caso clínico de una fractura subcapital de fémur secundaria a osteoporosis transitoria idiopática en una gestante a término.

\section{CASO CLÍNICO}

Gestante de 34 años, secundípara, sin antecedentes personales de importancia. Este embarazo cursó con anemia microcítica que requirió transfusión sanguínea en el tercer trimestre del embarazo. A las 38 semanas de gestación inicia cuadro clínico caracterizado por dolor intenso a nivel de ambas regiones inguinales, con incapacidad total para la deambulación, por lo se procede al ingreso hospitalario para estudio y tratamiento.

Se realiza radiografía de pelvis que es informa- 
da como normal (Figura 1), por lo que no se contraindica el parto vaginal. Al tercer día del ingreso, inicia trabajo de parto espontáneo con terminación mediante vacuum de silicona por agotamiento materno en el expulsivo. Se obtiene un recién nacido de sexo masculino de 3250 gramos, Apgar 9/10.

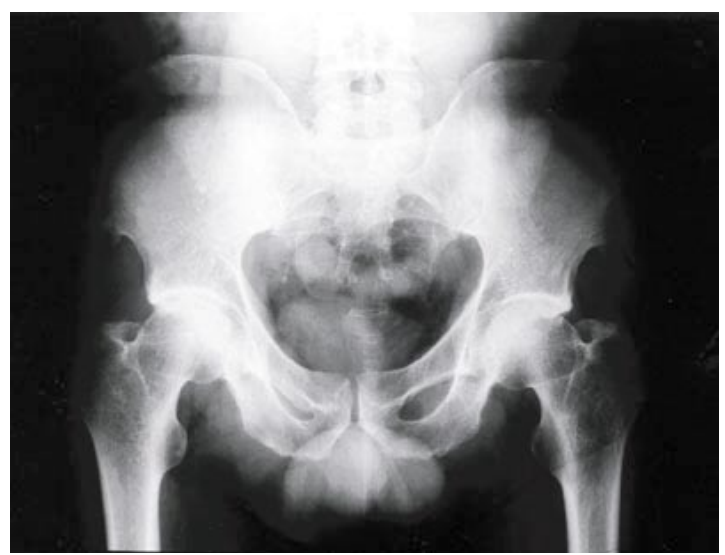

Figura 1. Radiografía de pelvis normal.

Durante el postparto, continua con la dificultad deambulatoria, por lo que es valorada por diferentes especialistas (neurología, traumatología y reumatología) realizándose como pruebas complementarias gammagrafía, TAC (tomografía axial computarizada) de columna, RNM (resonancia nuclear magnética) y EMG (electromiograma). EI EMG, TAC de columna y la gammagrafía son compatibles con la normalidad y la RNM (Figura 2) informa de fractura completa subcapital con deformidad de varo de la cadera izquierda, asociada a profusa edematización de la cabeza y cuello femoral, así como derrame sinovial. El traumatólogo aconseja reposo absoluto y tratamiento con calcitonina nasal, procediendo a su derivación a la Unidad de Metabolismo Óseo-Endocrinología para valorar etiología. En la misma se realiza DMO de columna y cadera informada como osteoporosis ( $T$ score columna $-2,8$, $T$ score cadera $-2,7$ ) (Figura 3 y 4). Se descarta posible origen tumoral o infeccioso, catalogándose como osteoporosis idiopática transitoria. Los niveles de calcemia, PTH (hormona paratiroídea), TSH (hormona estimulante del tiroides), osteocalcina y bioquímica, cuatro meses después del parto se situaban dentro de la normalidad, recomendándose tratamiento con calcio y risedronato. En la última evaluación clínica, tras diez meses, la paciente presenta mejoría clínica, con T score lumbar -2,9 y -2,1 femoral, por lo que continúa con el mismo tratamiento hasta la actualidad.

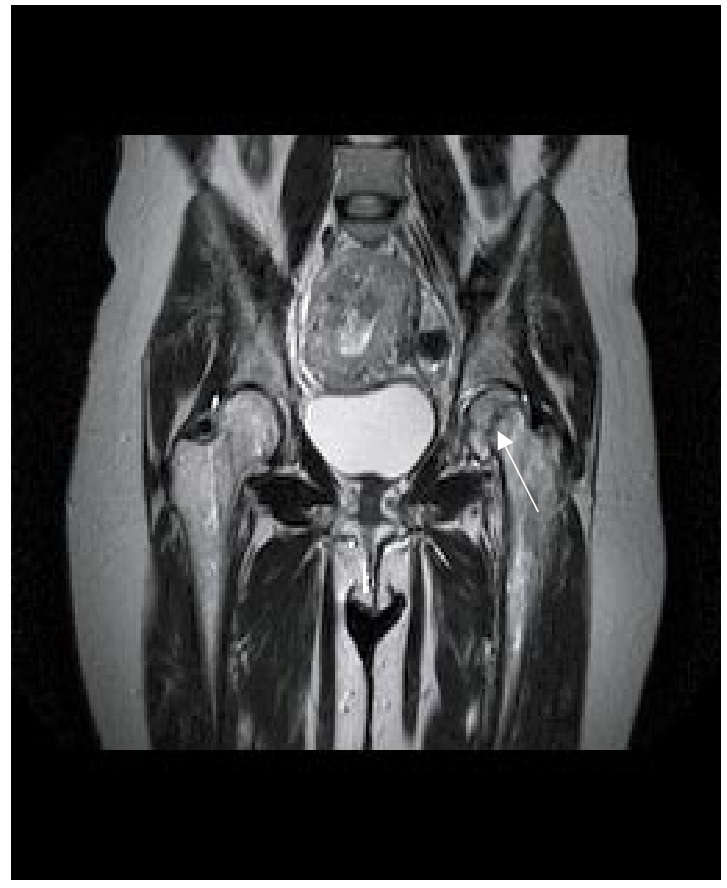

Figura 2. RNM de ambas caderas, flecha señala fractura subcapital.

\section{DISCUSIÓN}

Durante el embarazo, se produce una redistribución de la masa ósea, reduciéndose hasta un 3\% a nivel de columna lumbar y pelvis y un incremento en los huesos corticales (2). Se ha investigado la etiología de la osteoporosis secundaria, siendo hasta ahora desconocida; múltiples causas se han barajado: neurológicas, infecciosas, traumáticas, artríticas, neoplásicas (3), en nuestro caso, ninguna de éstas estaba presente.

La osteoporosis idiopática durante la gestación se presenta generalmente en el primer embarazo (al contrario de nuestro caso, con una primera gestación normal) en el curso del tercer trimestre o en el puerperio (4), tal como ocurrió en el caso presentado. Generalmente, las pruebas radiológicas se sitúan dentro de la normalidad (4) y son pruebas más específicas, como la RNM y la DMO, la que nos dan el diagnóstico clínico.

En muchos casos, se habla de una predisposición genética e incluso una osteoporosis asintomática manifestada en el tercer trimestre del embarazo, que es cuando se produce una mayor remodelación ósea y cambio en el metabolismo fosfo-cálcico, mostrada clínicamente con una fractura femoral. De hecho, el $60-80 \%$ del valor de la DMO está determinado por factores genéticos, existiendo una 


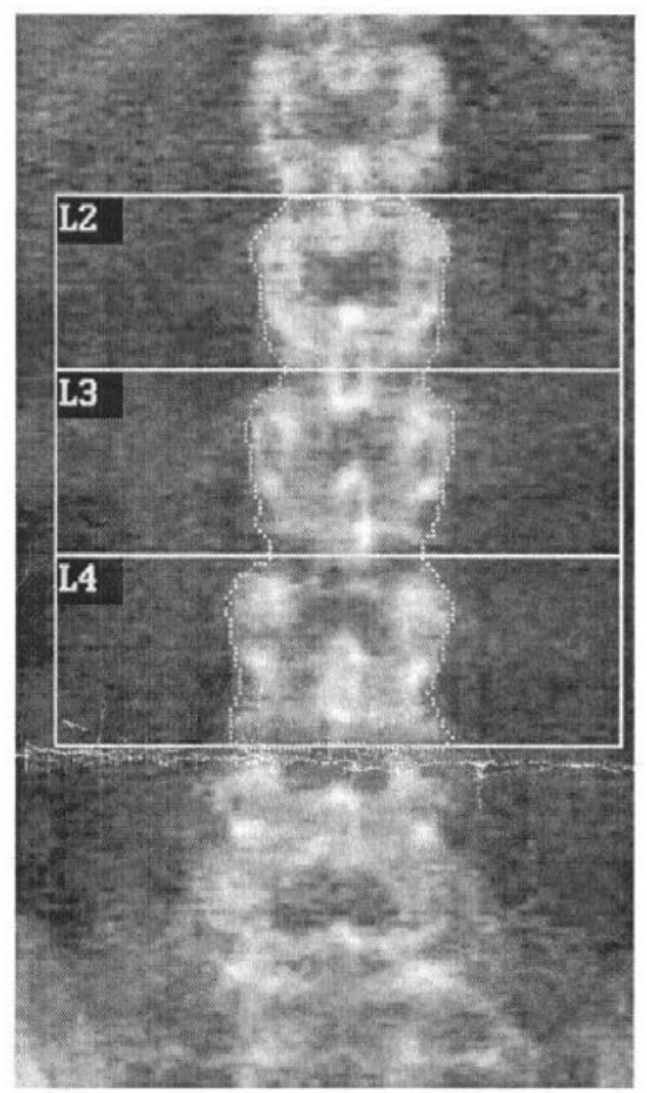

Image not for diagnostic use TOTAL BMD CV 1.0\%
DXA Scan Information:

Scan:

Scan Mode: $\quad$ Fast Array

Analysis: $\quad$ 3/29/07 11:00 - Ver 8.26

Operator: $\quad$ CRM

Model: $\quad$ Hologic QDR-4500W (S/N 49306)

Results Summary:

Total BMD: $\quad 0,765 \mathrm{~g} / \mathrm{cm}^{2}$

Peak reference: $\quad 71 \% \quad$ T score: -2.9

Age matched: $\quad 71 \% \quad$ Z score: -2.8

\begin{tabular}{lccccccc} 
Region & $\begin{array}{r}\text { Area } \\
{\left[\mathrm{cm}^{2}\right]}\end{array}$ & $\begin{array}{c}\text { BMC } \\
{[\mathrm{g}]}\end{array}$ & $\begin{array}{c}\text { BMD } \\
{\left[\mathrm{g} / \mathrm{cm}^{2}\right]}\end{array}$ & T score & $\% \mathrm{PR}$ & Z score & $\% A M$ \\
L2 & 11.44 & 8.29 & 0.725 & -2.8 & $71 \%$ & -2.7 & $71 \%$ \\
L3 & 12.98 & 10.03 & 0.773 & -2.8 & $71 \%$ & -2.8 & $72 \%$ \\
L4 & 15.37 & 12.13 & 0.790 & -3.0 & $71 \%$ & -2.9 & $71 \%$ \\
\hline & & & & & & & \\
Total: & 39.79 & 30.46 & 0.765 & -2.9 & $71 \%$ & -2.8 & $71 \%$
\end{tabular}

Figura 3. DMO de columna. predisposición genética a tener valores altos o bajos de densidad ósea (5).

En cuanto a los niveles hormonales, el calcio es el principal mineral implicado en la formación del esqueleto óseo. Durante le embarazo, se produce un descenso fisiológico del mismo y, en ocasiones, se ha visto que podría ser de beneficio su suplementación, aunque ésta no tiene efectos en los niveles de la DMO (2). Asimismo, los estrógenos son los más importantes reguladores del metabolismo óseo durante el embarazo, produciéndose un hipotético descenso de la DMO por los mismos en el tercer trimestre (5). No obstante, en nuestro caso todos los niveles hormonales se mantuvieron dentro de cifras normales para el puerperio.

Usualmente, los niveles de DMO vuelven a la normalidad transcurridos 6-12 meses postparto (5).
El tratamiento concomitante con bifosfonatos mejora los niveles de DMO, encontrándose hasta un incremento del 6\% a los 8-18 meses y del 9,5\% a los 2-4 años, frente a las que no los utilizaron (6). Ese tratamiento debe ser instaurado al término de la lactancia, ya que se ha visto su potencial teratógeno en animales de experimentación (7). En general, los resultados obtenidos a 5 años son mejores a nivel de columna que en cadera, y similares a los encontrados en pacientes postmenopáusicas (6).

En general, el riesgo de recurrencia en embarazos subsiguientes es bajo, pero no improbable por los factores genéticos asociados (6). Por todo lo anterior, es conveniente un seguimiento a largo plazo de estas pacientes mediante DMO para así poder prevenir eventuales fracturas. 


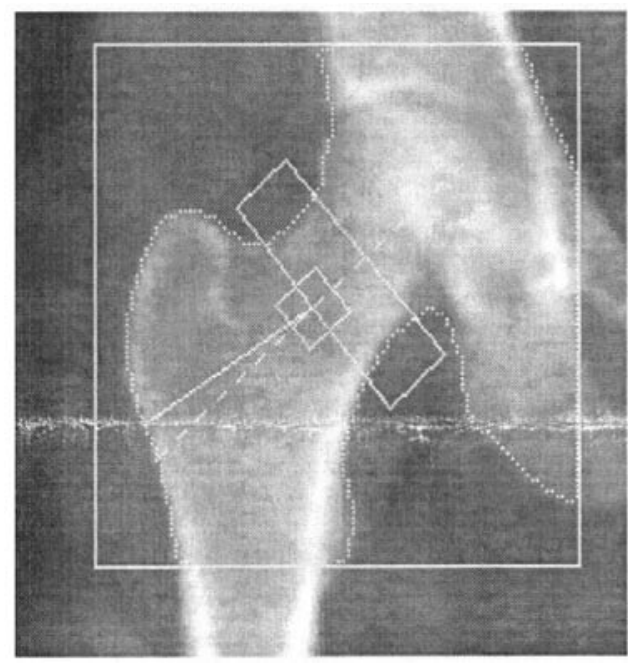

DXA Scan Information:

Scan: $\quad$ 3/29/07 - A0329070X

Scan Mode: $\quad$ Fast Array

Analysis: $\quad 3 / 29 / 0710: 58-$ Ver 8.26

Operator: $\quad$ CRM

Model: $\quad$ Hologic QDR-4500W (S/N 49306)

Results Summary:

Total [R]: $\quad 0,635 \mathrm{~g} / \mathrm{cm}^{2}$

Peak reference: $\quad 67 \% \quad$ T score: -2.5

Age matched: $\quad 68 \% \quad$ Z score: -2.4

\begin{tabular}{|c|c|c|c|c|c|c|c|}
\hline Region & $\begin{array}{l}\text { Area } \\
{\left[\mathrm{cm}^{2}\right]}\end{array}$ & $\begin{array}{c}\text { BMC } \\
\text { [g] }\end{array}$ & $\begin{array}{c}\mathrm{BMD}^{2} \\
{\left[\mathrm{~g} / \mathrm{cm}^{2}\right]}\end{array}$ & T score & $\% P R$ & Z score & $\% A M$ \\
\hline Neck & 4.56 & 2.82 & 0.617 & -2.1 & $73 \%$ & -1.9 & $74 \%$ \\
\hline Troch & 9.59 & 4.23 & 0.441 & -2.6 & $63 \%$ & -2.6 & $63 \%$ \\
\hline Inter & 15.11 & 11.55 & 0.764 & -2.2 & $69 \%$ & -2.2 & $69 \%$ \\
\hline ota & 29.27 & 18.60 & 0.635 & -2.5 & $67 \%$ & -2.4 & $68 \%$ \\
\hline Nard's & 1.2 & 0 & 0.458 & -2.4 & $62^{\circ}$ & -2.0 & 66 \\
\hline
\end{tabular}

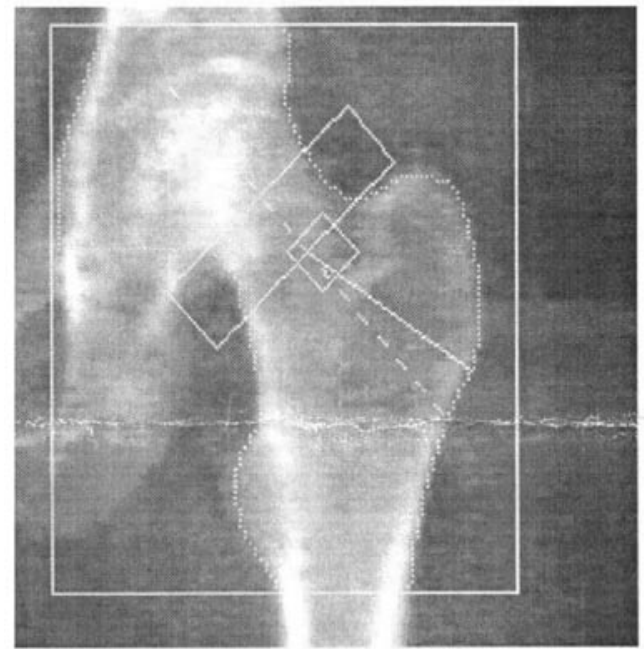

DXA Scan Information:

$\begin{array}{ll}\text { Scan: } & \text { 3/29/07 - A0329070X } \\ \text { Scan Mode: } & \text { Fast Array } \\ \text { Analysis: } & \text { 3/29/07 10:53 - Ver 8.26 } \\ \text { Operator: } & \text { CRM } \\ \text { Model: } & \text { Hologic QDR-4500W (S/N 49306) }\end{array}$

Results Summary:

$\begin{array}{lll}\text { Total [L] BMD: } & 0,599 \mathrm{~g} / \mathrm{cm}^{2} & \\ \text { Peak reference: } & 64 \% & \text { T score: }-2.8\end{array}$

Age matched: $\quad 64 \% \quad$ Z score: -2.7

Region Area BMC BMD T score \%PR Z score \%AM $\begin{array}{lccccccc} & {\left[\mathrm{cm}^{2}\right]} & {[\mathrm{g}]} & {\left[\mathrm{g} / \mathrm{cm}^{2}\right]} & & & & \\ \text { Neck } & 3.95 & 2.77 & 0.700 & -1.3 & 82 \% & -1.2 & 84 \% \\ \text { Troch } & 7.17 & 2.71 & 0.378 & -3.2 & 54 \% & -3.2 & 54 \%\end{array}$

$\begin{array}{lrrrrrrr}\text { Troch } & 7.17 & 2.71 & 0.378 & -3.2 & 54 \% & -3.2 & 54 \% \\ \text { Inter } & 19.34 & 12.76 & 0.660 & -2.8 & 60 \% & -2.8 & 60 \%\end{array}$

Figura 4. DMO ambas caderas

\section{BIBLIOGRAFÍA}

1. Cohen I, Melamed E, Lipkin A, Robinson D. Transient osteoporosis of pregnancy complicated by a pathologic subcapital hip fracture. J Trauma 2007;62(5):12813.

2. Oliveri B, Parisi MS, Zeni S, Mautalen C. Mineral and bone mass changes during pregnancy and lactation. Nutrition 2004;20(2):235-40.

3. Wood ML, Larson CM, Dahners LE. Late presentation of a displaced subcapital fracture of the hip in transient osteoporosis of pregnancy. J Orthop Trauma 2003;17(8):582-4.
4. Kabi F, Mkinsi O, Zrigui J. Pregnancy-associated osteoporosis. A new case. Rev Med Interne 2006;27(7):558-60.

5. Karlsson MK, Ahlborg HG, Karlsson C. Maternity and bone mineral density. Acta Orthop 2005;76(1):2-13.

6. O'sullivan SM, Grey AB, Singh R, Reid IR. Bisphosphonates in pregnancy and lactation-associated osteoporosis. Osteoporos Int 2006;17(7):1008-12.

7. Ornoy A, Wajnberg R, Diav-Citrin O. The outcome of pregnancy following pre-pregnancy or early pregnancy alendronate treatment. Reprod Toxicol 2006;22(4):578-9. 